\title{
Pepper-Pot Emittance Measurement of Laser-Plasma Wakefield Accelerated Electrons
}

\author{
R. P. Shanks, M. P. Anania, E. Brunetti, S. Cipiccia, B. Ersfeld, J. G. Gallacher, R. C. Issac, \\ M. R Islam, G. Vieux, G. H. Welsh, S. M. Wiggins, D. A. Jaroszynski \\ Department of Physics, Scottish Universities Physics Alliance, University of Strathclyde, \\ Glasgow G4 0NG, UK
}

\begin{abstract}
The transverse emittance is an important parameter governing the brightness of an electron beam. Here we present the first pepper-pot measurement of the transverse emittance for a mono-energetic electron beam from a laser-plasma wakefield accelerator, carried out on the Advanced Laser-Plasma High Energy Accelerators towards X-Rays (ALPHA-X) beam line. Mono-energetic electrons are passed through an array of $52 \mu \mathrm{m}$ diameter holes in a tungsten mask. The pepper-pot results set an upper limit for the normalised emittance at $5.5 \pm 1 \pi \mathrm{mm} \mathrm{mrad}$ for an $82 \mathrm{MeV}$ beam.
\end{abstract}

Keywords: wakefield acceleration, pepper pot, emittance, energy spread, laser-plasma interaction, relativistic electron beams

\section{INTRODUCTION}

Particle accelerators are at the forefront of scientific research and technology. One application for accelerators is as a source of tunable radiation with wavelengths varying from the infrared to X-ray regions of the electromagnetic spectra. However, conventional radiation sources, such as Diamond in the UK, are large scale facilities. Diamond is the largest scientific facility to be built in the UK in over 40 years. Current synchrotron light sources are around the size of five football fields and are extremely expensive. To be used widely as a research tool they must become much smaller and cheaper. Synchrotron light sources are used as diagnostic tools in many different areas of science and industry, such as: physics, biology, chemistry, pharmaceuticals, materials science, engineering, oil and gas. A laser-plasma wakefield accelerator ${ }^{1}$ combined with a magnetic undulator, can provide a source of synchrotron radiation from a device that is hundreds of times smaller and cheaper than conventional sources, and potentially with the capability of producing femtosecond duration pulses, as has been demonstrated in. ${ }^{2}$ Ultrashort light pulses are powerful tools for time-resolved studies of molecular and atomic dynamics.

Conventional accelerators use radio frequency $(\mathrm{RF})$ resonant cavities as accelerating structures. These are limited to $\sim 100 \mathrm{MV} / \mathrm{m}$ due to ionisation of the walls of the cavity and electron multipactor effect in the presence of high electric fields, and are typically restricted to around $20 \mathrm{MV} / \mathrm{m}$. The bunch duration of the electrons in these accelerators are relatively long, $>1 \mathrm{ps}$, limiting their application in ultra-fast physics. However, this can be overcome and the bunch duration reduced to $<100$ fs using magnetic compressors or electron bunch slicing techniques. ${ }^{3}$

Plasma, on the other hand, is already completely ionised and therefore can withstand much higher electric fields, in excess of $\sim 100 \mathrm{GV} / \mathrm{m}$, which is three orders of magnitude larger than conventional RF accelerators. ${ }^{4}$ Plasma as a medium for acceleration was first proposed by Tajima and Dawson in 1979. ${ }^{1}$ They proposed using plasma density waves that are driven by the ponderomotive force of a high intensity, ultra-short laser pulse. ${ }^{5}$ The plasma waves act as an accelerating structure, which are formed by the density wake of the laser pulse. The structure has dimensions of the order of the relativistic plasma wavelength, $\lambda_{p}=2 \pi c / \omega_{p}$. The wake travels at approximately the group velocity of the laser pulse, $v_{g} \simeq c\left(1-n_{e} / n_{c}\right)^{1 / 2}$, where $\omega_{p}=\left(e^{2} n_{e} / \gamma m_{0} \varepsilon_{0}\right)^{1 / 2}$ is

Further author information: (Send correspondence to:)

R. P. Shanks: E-mail: richard.shanks@strath.ac.uk

D. A. Jaroszynski: E-mail: d.a.jaroszynski@strath.ac.uk 
the relativistic plasma frequency, $n_{e}$ is the plasma density, $n_{c}=m \varepsilon_{0} \omega_{0}^{2} / e^{2}$ is the critical density, $\omega_{0}$ is the laser frequency, and $c$ is the speed of light in vacuum. When the plasma wave becomes relativistic the electrons are self injected into the rear of the accelerating structure and accelerate to relativistic velocities, ${ }^{5}$ only limited by the group velocity of the laser pulse due to de-phasing. The distance the electron can propagate within the accelerator is known as the dephasing length, $\mathrm{L}_{d}$, for a weakly relativistic laser pulse is given by, $L_{d}=c \omega_{0}^{2} / \omega_{p}^{3}$ where $\gamma=\left(1+a_{0}^{2}\right)^{1 / 2}$ is the relativistic factor of the plasma electrons. For $a_{0}>1$ the wake is elongated by a factor of $(4 / 3) \sqrt{a_{0}} .6$ However, the dephasing length can be increased by decreasing the plasma density, which in turn decreases the plasma frequency. As the electrons are accelerated by the potential, they acquire energy from the wave and thus damp the amplitude of the wake. ${ }^{7}$

The increased accelerating field reduces the required distance to reach high energies. For example, it would take several meters to obtain $50 \mathrm{MeV}$ electrons in a conventional accelerator, but with a plasma accelerator this can be achieved in a $2 \mathrm{~mm}$ gas jet. ${ }^{8-10}$ The laser pulse filaments across the gas jet to create acceleration distances several $\mathrm{mm}$ long. To reach high energies in plasma accelerators it is essential to guide the laser pulse in plasma to increase the interaction length. This has been successfully achieved in a discharge capillary waveguide (used to guide the laser pulse over a much greater distance than its Rayleigh length) where $1 \mathrm{GeV}$ electrons have been produced in a $3 \mathrm{~cm}$ structure. ${ }^{11}$ However, $\mathrm{RF}$ accelerator technology has reached a high level of maturity with proven success and therefore plasma accelerators require significant development to be competitive.

The laser wakefield accelerator (LWFA) has a significant advantage over conventional accelerators due to their inherent properties. One such property is the electron bunch duration is predicted to be of the order $10 \mathrm{fs},{ }^{12}$ given by the short scale length of the accelerator for typical densities of $n_{p} \approx 10^{18}-10^{19} \mathrm{~cm}^{-3}$. The short bunch duration and high charge $(>10 \mathrm{pC})^{8-10}$ should allow peak currents $\geq 1 \mathrm{kA}$ to be produced. A low emittance is also expected because of the very small transverse dimensions of the electron bunches.

These properties make the LWFA electron beams an attractive driver of undulator radiation. The undulator radiation is given by:

$$
\lambda=\frac{\lambda_{u}}{2 h \gamma^{2}}\left(1+\frac{a_{u}^{2}}{2}+\gamma^{2} \vartheta^{2}\right)
$$

where $\lambda_{u}$ is the undulator period, $\mathrm{h}$ is the harmonic number, $\gamma$ is the Lorentz factor $a_{u}$ is the undulator parameter and $\vartheta$ is the angle of radiation relative to the electron axis. The radiation emitted is dominated by the $\lambda_{u} / 2 h \gamma^{2}$ term, which is simply a double Doppler contraction of the undulator period. The radiation wavelength is extended by the $\left(1+a_{u}^{2} / 2\right)$ term due the periodic deviation of the electrons as a result of the periodic magnetic field of the undulator. ${ }^{13}$ The wavelength emitted also increases with angle as described by $\gamma^{2} \vartheta^{2}$ term. ${ }^{13}$ A low emittance maximises the betatron function, $\beta=\pi r_{0}^{2} / \varepsilon,{ }^{14}$ which is a measure of the distance over which the minimum focal spot of the electrons can be kept. An increased betatron function decreases the the angular spread of radiation produced from the undulator which increases the monochromaticity of the radiation. It is therefore essential that the emittance is a low as possible.

The LWFA could also provide a convenient and compact driver of a free electron laser (FEL), which could be a disruptive technology. The high peak current and low emittance of the electron beams will maximise the FEL gain parameter, $\rho$, and thus minimise the gain length required for a self amplified spontaneous emission (SASE) FEL. ${ }^{15}$

\section{TRANSVERSE EMITTANCE MEASUREMENT}

In this section we present the latest developments from the ALPHA-X project at the University of Strathclyde. The ALPHA-X project beam line has a high resolution electron spectrometer, which enables the energy spread to be measured with very high resolution. Furthermore, a pepper-pot emittance measurement system has been set-up to determine the emittance. 


\subsection{Experimental Setup}

The experimental setup at Strathclyde is shown in Figure $1 .{ }^{16}$ The TOPS ${ }^{17}$ laser system produces a $900 \mathrm{~mJ}$ (on target), 35 fs laser pulse with a central wavelength of $800 \mathrm{~nm}$, focused to a $20 \mu \mathrm{m}\left(1 / e^{2}\right.$ radius) spot with a $\mathrm{F} / 16$ spherical mirror. The peak intensity is $I=2 \times 10^{18} \mathrm{Wcm}^{-2}$ (above that needed for self injection) and the normalised vector potential $a_{0}=e E /\left(m_{0} c \omega_{0}\right) \approx 1.2, E=\left(2 I / c \varepsilon_{0}\right)^{1 / 2}$ is the peak electric field of the laser pulse. A $2 \mathrm{~mm}$ diameter gas jet nozzle is used as a plasma medium and is set to a plasma density of $n_{e}=3 \times 10^{19} \mathrm{~cm}^{-3}$.

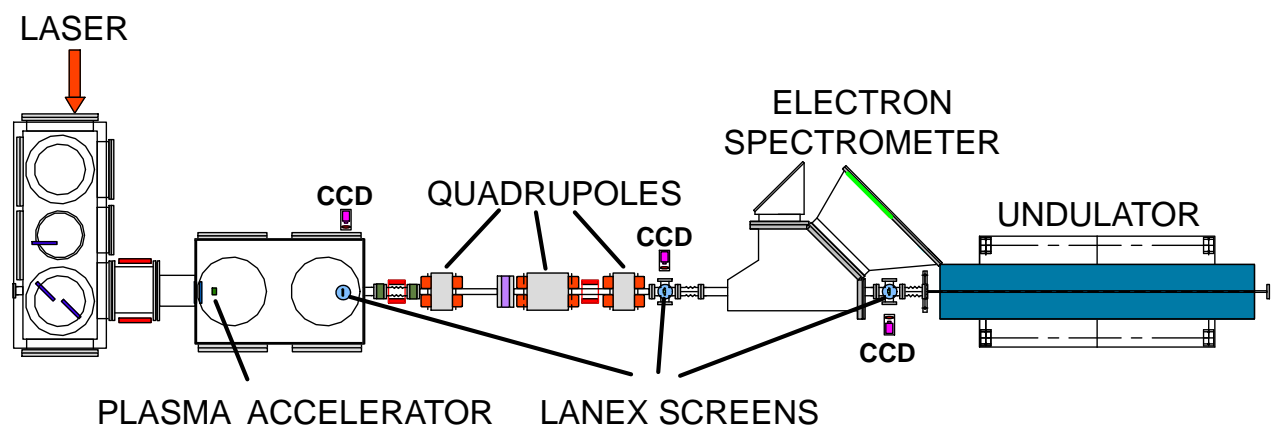

Figure 1. ALPHA-X experimental set-up: a high powered laser is focused onto a gas jet to produce mono-energetic electrons. The electrons are imaged on three LANEX targets and focused into an electron spectrometer and magnetic undulator by a quadrupole triplet magnetic lens.

Electrons are detected on the $1^{\text {st }}$ LANEX screen, as shown in Figure 1, which is placed 45 degrees to the electron beam axis and then imaged with a CCD camera. The electrons propagate through a triplet of focusing quadrupoles magnets. The electrons are also imaged on the $2^{\text {nd }}$ and $3^{\text {rd }}$ pop-in LANEX screens placed at the entrance to the electron spectrometer and undulator respectively.

The electron energy is measured by an electron spectrometer, which consists of an electromagnet that can be switched on to bend the electrons out of the beam line. The maximum magnetic field of the spectrometer is $1.7 \mathrm{~T}$, which allows energy measurements up to $600 \mathrm{MeV}$. The spectrometer utilises the Browne-Buechner design ${ }^{18}$ to focus the electrons on both the horizontal and vertical planes over a wide energy range. This allows high resolution spectra to be measured, providing $0.1 \%$ energy spread for $100 \mathrm{MeV}$ electrons. Electrons are detected on a Ce:YAG crystal that is imaged using a 12 bit CCD camera. In addition, the quadrupoles magnets can be used to optimise the system.

\subsection{Energy Measurement}

The electron trajectory and wakefield accelerator is optimised on the $1^{\text {st }}$ LANEX target to produce a low divergence beam with a good shot-to-shot pointing stability. ${ }^{19}$ Figure 2 displays an optimised beam with a divergence of $1.5 \mathrm{mrad}(\mathrm{FWHM})$. By using the quadrupoles it is possible to optimise the electrons entering the electron spectrometer to produce narrow energy spread, high resolution electron spectra. Figure 3(b) shows 5

high resolution images. The 5 shots have an average energy of $82 \pm 4 \mathrm{MeV}$ and an r.m.s. energy spread of $1.1 \pm 0.4 \%$. The normalised emittance calculated later will use this average energy value. The highest energy obtained was $89 \mathrm{MeV}$ and the lowest relative r.m.s. energy spread was $\sigma_{\gamma} / \gamma=0.79 \%$, as shown in Figure 3(a). This energy represents the lowest energy spread ever measured from a LWFA. ${ }^{20}$

\subsection{Emittance Measurement}

Emittance is a measure of the beam quality and determines the parallelism of the beam and thus allows us to compare the quality of beams for application.

The pepper-pot emittance measurement technique is preferable for LWFA electrons because it is single shot and not prone to space charge effects. This technique uses a mask to block most of the electron beam and 


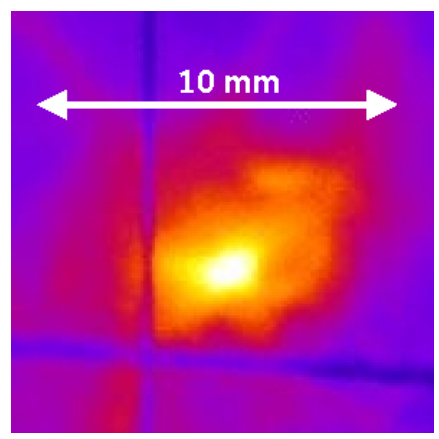

Figure 2. Mono-energetic electron beam of $1.5 \mathrm{mrad}(\mathrm{FWHM})$ divergence, imaged on the first LANEX screen. (False colour added for clarity.)

(a)

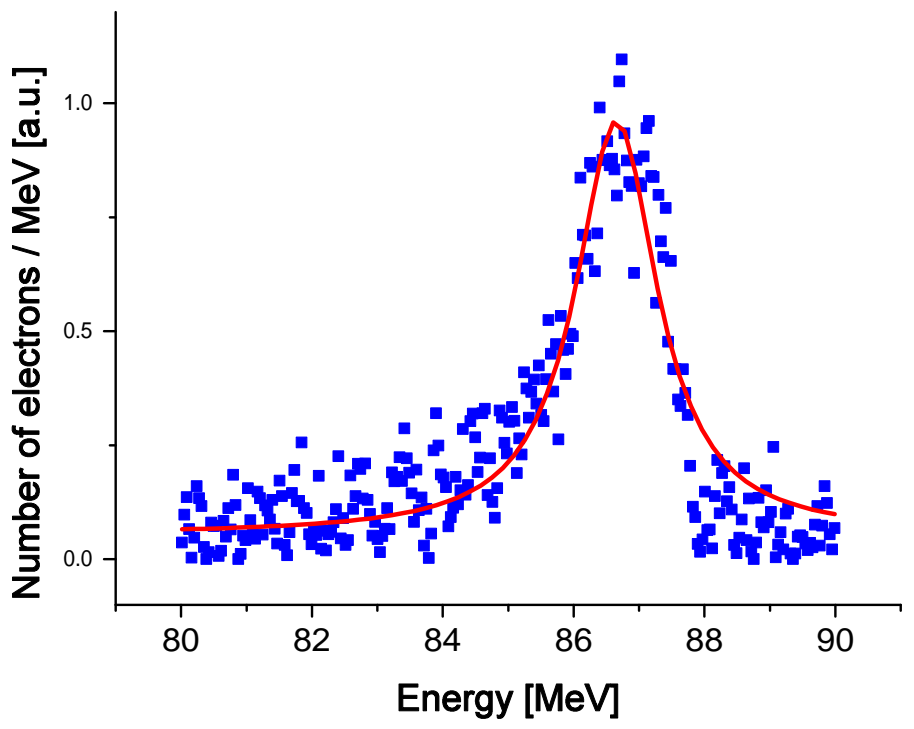

(b)

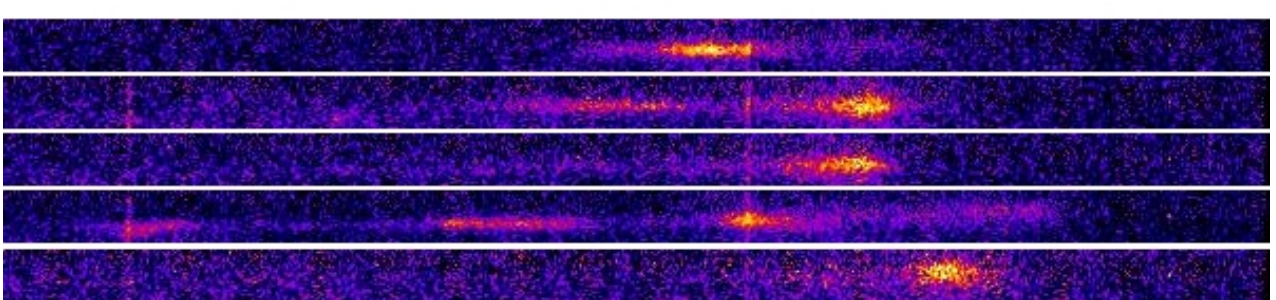

Figure 3. (a) An electron energy spectrum with a peak energy of $89.2 \mathrm{MeV}$ and a $\sigma_{\gamma} / \gamma=0.8 \%$. (b) Five false colour images of the electron spectra with quadrupole focusing to produce high resolution electron spectra. The energy range shown is $60-100 \mathrm{MeV}$ (left to right) and the averge energy is $82 \pm 4 \mathrm{MeV}$. (a) is the plot of the last spectra in (b).

only transmit small beamlets. The beam remains unfocused and therefore experiences little space charge effect. Other techniques, such a the quadrupole scan, which scans the focal spot of an electron beam by varying the quadrupole focal length ${ }^{21}$ are more susceptible to space charge effects. Shot-to-shot variations in the emittance can also be monitored as the pepper pot technique is a single shot measurement.

Pepper-pot emittance measurements are an established tool for diagnosing RF photo-injectors. ${ }^{22}$ The relatively low energy, space charge dominated beams are well suited to this type of measurement. A previous 
emittance measurement was performed on low energy Maxwellian distribution LWFA electrons beams. ${ }^{23}$ However, in that experiment the electron beam passed through a stainless steel collimator to remove high divergence electrons to measure a low emittance of only part of the cleaned beam. In this section we present the first single shot measurement of the transverse emittance for complete, mono-energetic LWFA electron beams.

The r.m.s. emittance is defined as the correlation between the space, $x$, and the transverse momentum, $x^{\prime}$, of the electron beam. It is also the r.m.s. area encompassed by the beam in $2 \mathrm{D}$ phase-space $\left(x, x^{\prime}\right)^{14}$

$$
\varepsilon_{x, r m s}=\sqrt{\left.<x^{2}><x^{\prime 2}>-<x x^{\prime}\right\rangle^{2}}
$$

However, it is possible to measure the emittance directly from the pepper-pot beamlets as shown by Zhang ${ }^{24}$

$$
\varepsilon_{x, r m s}^{2}=\frac{1}{N^{2}}\left\{\left[\sum_{j=1}^{p} n_{j}\left(x_{j}-\bar{x}\right)^{2}\right]\left[\sum_{j=1}^{p}\left[n_{j} \sigma_{x_{j}^{\prime}}^{\prime 2}+n_{j}\left(x_{j}^{\prime}-\bar{x}^{\prime}\right)^{2}\right]\right]-\left[\sum_{j=1}^{p} n_{j} x_{j} x_{j}^{\prime}-N \bar{x} \bar{x}^{\prime}\right]\right\},
$$

where $N=\sum_{j=1}^{p} n_{j} ; n_{j}$ is the number of electrons passing through the $\mathrm{j}^{\text {th }}$ hole, $x_{j}$ is the $\mathrm{j}^{\text {th }}$ hole position, $\bar{x}=\left(\sum_{j=1}^{p} n_{j} x_{j} / N\right)$ is the mean hole position, $\sigma_{x_{j}^{\prime}}^{\prime}=\sigma_{x_{j}^{\prime}} / L_{m d}$ is the r.m.s. divergence of the $\mathrm{j}^{\text {th }}$ beamlet, $x_{j}^{\prime}=\left(X_{j}-x_{j}\right) / L_{m d}$ is the divergence of the $\mathrm{j}^{\text {th }}$ beamlet, $X_{j}$ is the peak position of the detected $\mathrm{j}^{\text {th }}$ beamlet, $\bar{x}^{\prime}$ is the mean divergence of all the beamlets, $L_{m d}$ is the distance from the mask to the detector and p is the total number of beamlets.

The mask consists of an array of $11 \times 11$ holes drilled in tungsten. A slight taper of the hole gives an effective hole diameter of $52 \pm 7 \mu \mathrm{m}$. The pepper-pot mask is mounted on a rotation stage to allow it to be moved in and out of the beam line, because the measurement is destructive and scatters the electrons. The mask is aligned using a HeNe (which is co-aligned with the beam line). The transmitted and back reflected light is used to ensure that the mask is placed normal to the electron beam.

The electrons are measured and optimised on the first LANEX screen, as before. The pepper-pot mask is then rotated into the beam and the transmitted electrons measured on the screen. Figure 4 (a) and 5 (a) shows a typical pepper-pot measurement, with false colour added for clarity.

To obtain $\sigma_{j}^{\prime}$ directly form the image, the mask hole radius, $\mathrm{r}$, should be small compared with the measured spot radius, $\sigma_{j} . \sigma_{j}^{\prime}$ is over estimated in this measurement and the mask hole size must be taken into consideration. As a rough approximation, the mask hole size is subtracted from the measured spot size before $\sigma_{j}^{\prime}$ is calculated. The hole size is also magnified because the electron beam is diverging and $\sigma_{j}^{\prime}$ is given by

$$
\sigma_{j, \text { corrected }}^{\prime}=\frac{\left(\sigma_{j}-d M\right)}{L_{m d}}
$$

where $M=\left(L_{g m}+L_{m d}\right) / L_{m d}$, is a magnification factor given by a ratio of the distance from the gas jet to the mask $\left(L_{g m}\right)$ and the mask to the detector $\left(L_{m d}\right)$. The mask is near the mid point between the gas jet and the detector such that $\mathrm{M}=2.05$.

The normalised emittance, $\varepsilon_{x, r m s}^{n}$, for the beams is $5.5 \pm 1 \pi \mathrm{mm} \mathrm{mrad}$ and $7.8 \pm 1 \pi \mathrm{mm}$ mrad for Figures 4 and 5 respectively, assuming $\mathrm{E}=82 \mathrm{MeV}$. The errors arise from the uncertainty in the electron energy and the taper inside the mask's hole. By decreasing this taper and measuring the electron energy simultaneously with the emittance, it should be possible to reduce this error. The measured beamlet width is also increased by the resolution limit of the detector which has not been corrected here. Therefore, this measurement places an upper limit to the measured emittance of the mono-energetic electrons.

It is possible to use this upper limit of the emittance to set an upper limit of the source size because the emittance is conserved as the beam diverges. Figure 6 shows the source as a function of transverse emittance for a beam with the same divergence ( $4 \mathrm{mrad}$ FWHM) as in Figure 4. The upper limit of the emittance sets a maximum source size of $18 \mu \mathrm{m}$. However, it is know from simulations that the source size is smaller than 2 $\mu \mathrm{m}$. As can be seen in Figure 6, a beam with a divergence of $4 \mathrm{mrad}$ would have an emittance of $<0.5 \pi \mathrm{mm}$ 
(a)

(b)

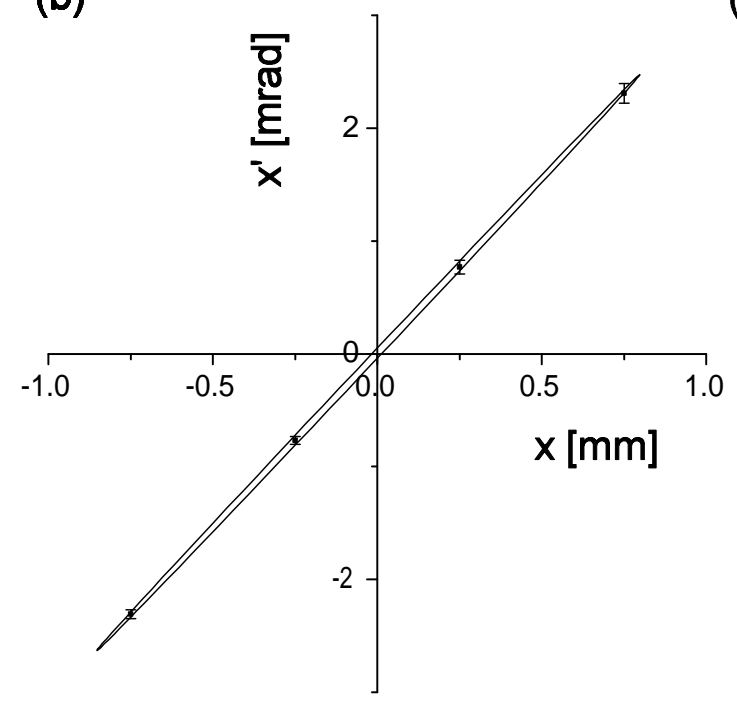

(c)

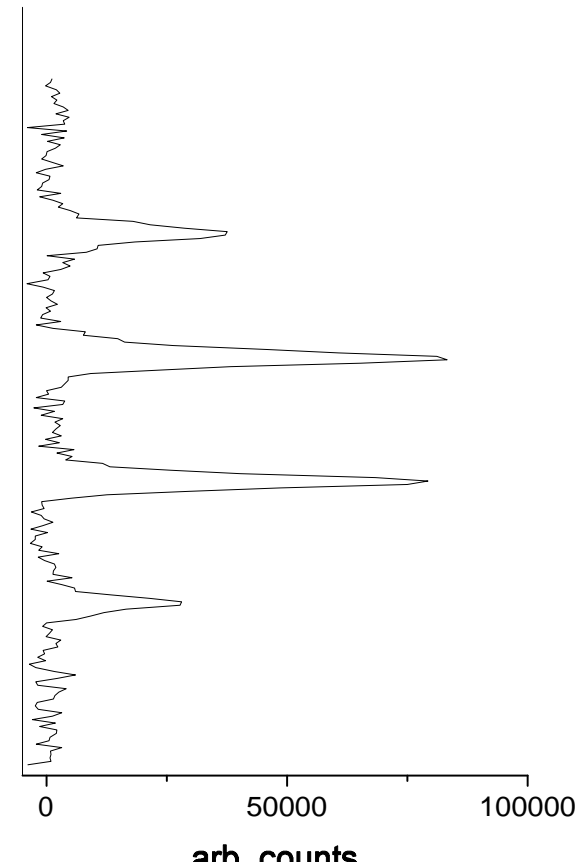

arb. counts

Figure 4. ((a) Image from a pepper-pot emittance measurement for a 4 mrad divergence beam. The normalised r.m.s. emittance (corrected for hole size and for $82 \mathrm{MeV}$ electrons) is $5.5 \pm 1 \pi \mathrm{mm}$ mrad. (b) shows the phase-space diagram and (c) charge distribution across the electron bunch. 


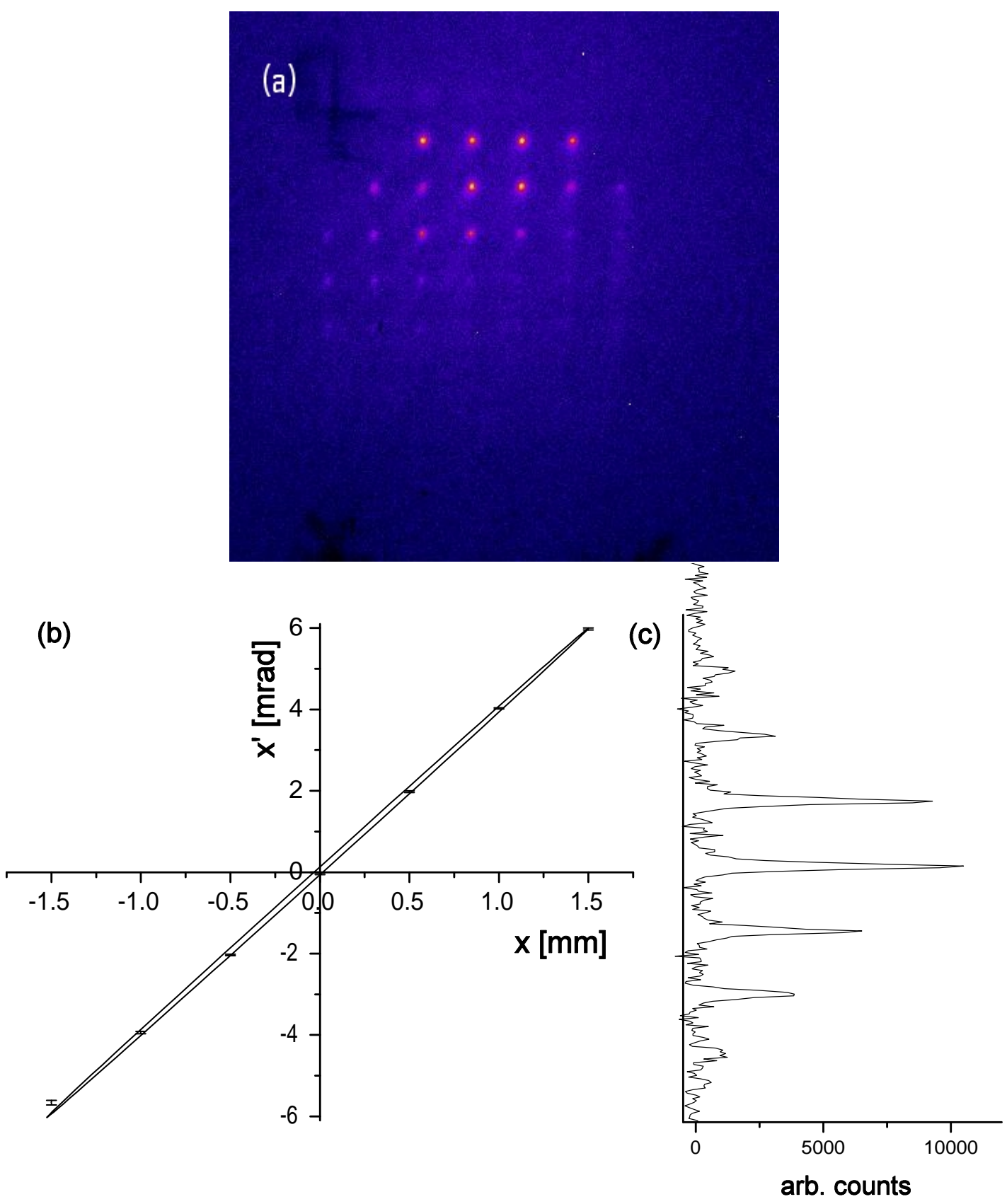

Figure 5. (a) Image from a pepper-pot emittance measurement for a 10 mrad divergence beam. The normalised r.m.s. emittance (corrected for hole size and for $82 \mathrm{MeV}$ electrons) is $7.8 \pm 1 \pi \mathrm{mm}$ mrad. (b) shows the phase-space diagram and (c) charge distribution across the electron bunch.

mrad. These values of the source as a function of emittance were calculated using General Particle Tracer (GTP) code. ${ }^{25,26}$ GPT considers space charge effect when tracing the electron beam. In the simulations, a charge of $100 \mathrm{pC}$, energy spread $\sigma_{\gamma} / \gamma=1 \%$ and a $10 \mathrm{fs}$ bunch duration were assumed.

It is possible that the emittance for LWFA electron beams could be as low a $0.1 \pi \mathrm{mm}$ mrad, because beams with divergence as low as $1.5 \mathrm{mrad}(\mathrm{FWHM})$ have been observed in our measurements. In Figure 7, GTP is 


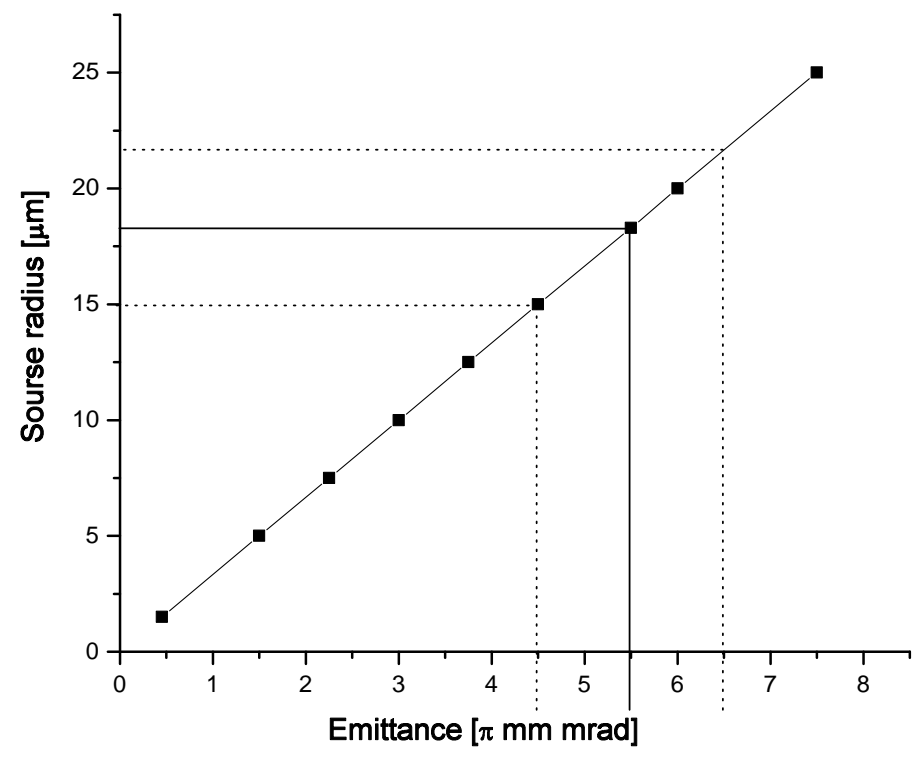

Figure 6. Source size as a function of emittance for a $4 \mathrm{mrad}$ beam as calculated by GTP (assumed beam parameters $100 \mathrm{pC}$ charge, $10 \mathrm{fs}$ bunch duration and $\sigma_{\gamma} / \gamma=1 \%$.)

used to show that space charge does not destroy the low beam emittance. For emittance $>1 \pi \mathrm{mm}$ mrad, space charge has little effect. The effect is greater for a beam of $0.1 \pi \mathrm{mm}$ mrad. However, the emittance only increases to $0.5 \pi \mathrm{mm}$ mrad in the first meter of propagation.

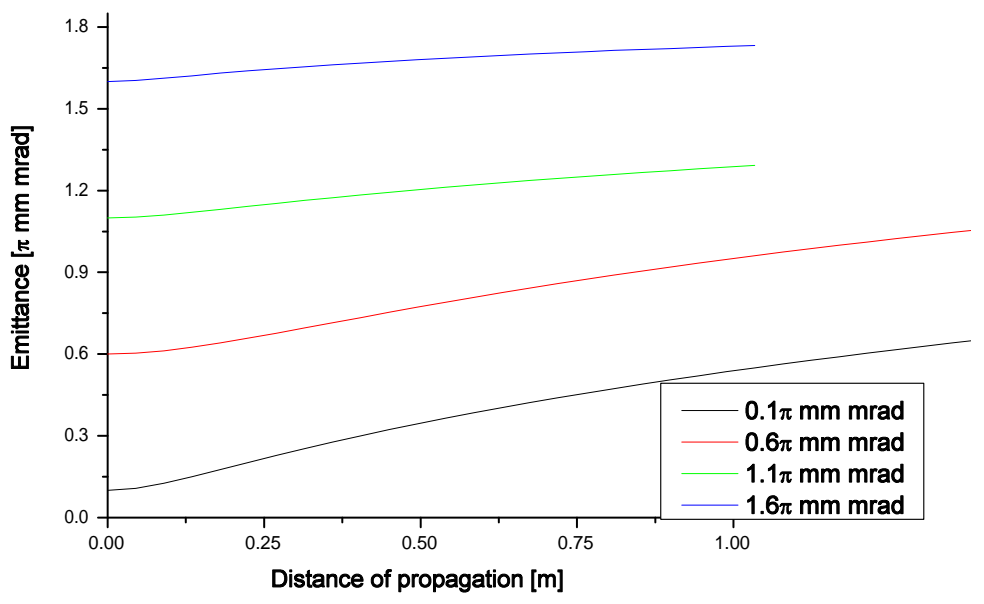

Figure 7. Emittance growth during the electron beam's propagation after the accelerator, calculated using GPT. show's emittance is not destroyed by space charge effects. (Assumed beam parameters of $100 \mathrm{pC}$ charge, $10 \mathrm{fs}$ bunch duration, $2 \mu \mathrm{m}$ source size and $\sigma_{\gamma} / \gamma=1 \%$.) 


\section{CONCLUSION}

This paper demonstrates the first pepper-pot measurement of the transverse emittance of mono-energetic electrons from LWFA. The results confirm an upper limit for the emittance of $5.5 \pm 1 \pi \mathrm{mm}$ mrad, set by the resolution limit of the detector. GPT simulations show that the source size for the beam measured is less that $18 \mu \mathrm{m}$. However, a source size of $2 \mu \mathrm{m}$, predicted from simulations, reduces the emittance to $<0.5 \pi \mathrm{mm} \mathrm{mrad}$. These low emittance, small source size, high current beams should be very useful in future applications as they are not destroyed by space charge effect.

Experiments using high resolution electron spectrometer confirmed the r.m.s. spread to be as low as $0.8 \%$ for electrons in the $85-90 \mathrm{MeV}$ energy range. This is an upper limit to the energy spread and further optimisation of the system and deconvolution could result in lower measured energy spread electrons. These results are important for future applications of electron beams such as a driving source for SASE FELs, ${ }^{27}$ which require low energy spread, low emittance beams.

\section{ACKNOWLEDGMENTS}

We acknowledge the support of the U. K. EPSRC, the European Community - New and Emerging Science and Technology Activity under the FP6 "Structuring the European Research Area" programme (project EuroLEAP, contract number 028514), the Laserlab-Europe consortium and the Extreme Light Infrastructure (ELI) project.

\section{REFERENCES}

[1] T. Tajima and J. M. Dawson, "Laser electron-accelerator," Physical Review Letters 43(4), pp. 267-270, 1979.

[2] H. P. Schlenvoigt, K. Haupt, A. Debus, F. Budde, O. Jackel, S. Pfotenhauer, H. Schwoerer, E. Rohwer, J. G. Gallacher, E. Brunetti, R. P. Shanks, S. M. Wiggins, and D. A. Jaroszynski, "A compact synchrotron radiation source driven by a laser-plasma wakefield accelerator," Nature Physics 4(2), pp. 130-133, 2008.

[3] S. Khan, K. Holldack, T. Kachel, R. Mitzner, and T. Quast, "Femtosecond undulator radiation from sliced electron bunches," Physical Review Letters 97(7), 2006.

[4] E. Esarey, P. Sprangle, J. Krall, and A. Ting, "Overview of plasma-based accelerator concepts," Ieee Transactions on Plasma Science 24(2), pp. 252-288, 1996.

[5] T. Katsouleas, "Accelerator physics - electrons hang ten on laser wake," Nature 431(7008), pp. 515-516, 2004.

[6] W. Lu, M. Tzoufras, C. Joshi, F. S. Tsung, W. B. Mori, J. Vieira, R. A. Fonseca, and L. O. Silva, "Generating multi-gev electron bunches using single stage laser wakefield acceleration in a $3 \mathrm{~d}$ nonlinear regime," Physical Review Special Topics-Accelerators and Beams 10(6), 2007.

[7] A. J. W. Reitsma, R. A. Cairns, R. Bingham, and D. A. Jaroszynski, "Efficiency and energy spread in laser-wakefield acceleration," Physical Review Letters 94(8), 2005.

[8] C. G. R. Geddes, C. Toth, J. van Tilborg, E. Esarey, C. B. Schroeder, D. Bruhwiler, C. Nieter, J. Cary, and W. P. Leemans, "High-quality electron beams from a laser wakefield accelerator using plasma-channel guiding," Nature 431(7008), pp. 538-541, 2004.

[9] S. P. D. Mangles, C. D. Murphy, Z. Najmudin, A. G. R. Thomas, J. L. Collier, A. E. Dangor, E. J. Divall, P. S. Foster, J. G. Gallacher, C. J. Hooker, D. A. Jaroszynski, A. J. Langley, W. B. Mori, P. A. Norreys, F. S. Tsung, R. Viskup, B. R. Walton, and K. Krushelnick, "Monoenergetic beams of relativistic electrons from intense laser-plasma interactions," Nature 431(7008), pp. 535-538, 2004.

[10] J. Faure, Y. Glinec, A. Pukhov, S. Kiselev, S. Gordienko, E. Lefebvre, J. P. Rousseau, F. Burgy, and V. Malka, "A laser-plasma accelerator producing monoenergetic electron beams," Nature 431(7008), pp. 541-544, 2004.

[11] W. P. Leemans, B. Nagler, A. J. Gonsalves, C. Toth, K. Nakamura, C. G. R. Geddes, E. Esarey, C. B. Schroeder, and S. M. Hooker, "Gev electron beams from a centimetre-scale accelerator," Nature Physics 2(10), pp. 696-699, 2006.

[12] A. Pukhov and J. Meyer-ter Vehn, "Laser wake field acceleration: the highly non-linear broken-wave regime," Applied Physics B-Lasers and Optics 74(4-5), pp. 355-361, 2002. 
[13] P. Luchini and H. Motz, Undulators and Free-Electron Lasers, Oxford Science Publications, 1990.

[14] S. Humphries, Charged Particle Beams, John Wiley and Sons, 1990.

[15] R. Bonifacio, C. Pellegrini, and L. M. Narducci, "Collective instabilities and high-gain regime in a freeelectron laser," Optics Communications 50(6), pp. 373-378, 1984.

[16] D. A. Jaroszynski, R. Bingham, E. Brunetti, B. Ersfeld, J. Gallacher, B. van der Geer, R. Issac, S. P. Jamison, D. Jones, M. de Loos, A. Lyachev, V. Pavlov, A. Reitsma, Y. Saveliev, G. Vieux, and S. M. Wiggins, "Radiation sources based on laserplasma interactions," Philosophical Transacitons of the Royal Society A 364, pp. 689-710, 2006.

[17] D. A. Jaroszynski, B. Ersfeld, G. Giraud, S. Jamison, D. R. Jones, R. C. Issac, B. M. W. McNeil, A. D. R. Phelps, G. R. M. Robb, H. Sandison, G. Vieux, S. M. Wiggins, and K. Wynne, "The strathclyde terahertz to optical pulse source (tops)," Nuclear Instruments and Methods in Physics Research Section a-Accelerators Spectrometers Detectors and Associated Equipment 445(1-3), pp. 317-319, 2000.

[18] C. P. Browne and W. W. Buechner, "Broad-range magnetic spectrograph," The Review of Scientific Instruments 27(11), pp. 899-907, 1956.

[19] R. C. Issac, G. Vieux, G. Welsh, R. Shanks, E. Brunetti, S. Cipiccia, M. P. Anania, X. Yang, M. S. Wiggins, M. R. Islam, B. Ersfeld, J. Farmer, G. Raj, S. Chen, D. Clark, T. McCanny, and D. A. Jaroszynski, "Electron beam pointing stability of a laser wakefield accelerator," Proc. SPIE 7359 This Volume, 2009.

[20] S. M. Wiggins, M. P. Anania, E. Brunetti, S. Cipiccia, B. Ersfeld, M. R. Islam, R. C. Issac, G. Raj, R. P. Shanks, G. Vieux, G. H. Welsh, W. A. Gillespie, A. M. MacLeod, and D. A. Jaroszynski, "Narrow spread electron beams from a laser-plasma wakefield accelerator.," Proc. SPIE 7359 This Volume, 2009.

[21] B. E. Carlsten, "Characterizing the emittance contribution due to rotated quadrupoles and canonical angular momentum using the quadrupole scan technique in electron accelerators," Review of Scientific Instruments 70(3), pp. 1672-1683, 1999.

[22] S. G. Anderson, J. B. Rosenzweig, G. P. LeSage, and J. K. Crane, "Space-charge effects in high brightness electron beam emittance measurements," Physical Review Special Topics-Accelerators and Beams 5(1), 2002.

[23] S. Fritzler, E. Lefebvre, V. Malka, F. Burgy, A. E. Dangor, K. Krushelnick, S. P. D. Mangles, Z. Najmudin, J. P. Rousseau, and B. Walton, "Emittance measurements of a laser-wakefield-accelerated electron beam," Physical Review Letters 92(16), 2004.

[24] M. Zhang, "Emittance formula for slits and pepper-pot measurement," Tech. Rep. FERMILAB-TM-1998, 1998.

[25] B. Van Der Geer and M. Loos, General Particle Tracer code: design, implementation and application. PhD thesis, 2001.

[26] B. Van Der Geer and M. Loos, The general Particle Tracer. PhD thesis, 2001.

[27] M. Anania, D. Clark, S. van der Geerb, M. de Loos, R. Isaac, G. Reitsma, A.J.W.and Welsh, W. S.M., and D. Jaroszynski, "Transport of ultra-short electron bunches in a free-electron laser driven by a laser-plasma wakefield accelerator," Proc. SPIE 7359 This Volume, 2009. 